\title{
CYTOLOGICAL PREPARATIONS FROM PROTEIN-FREE FLUIDS
}

\author{
BY \\ M. E. A. POWELL \\ From King's College Hospital Medical School, London
}

(RECEIVED FOR PUBLICATION JULY 6, 1959)

It is necessary for the preparation of stained smears for cytological examination that cells be made to adhere to glass slides. From sites such as the vagina, cervix, oral cavity, and bronchial tree this is a simple matter, as the fluid portion of the specimen contains sufficient protein material to induce good adhesion to the slide when treated with a suitable fixative. If the fluid portion of the specimen is protein-free it is much more difficult to persuade the cells to stick to the slide, many of them floating off when the slide is placed in the fixative. For this reason the cytological examination of urine, and to a lesser extent of pleural fluid, has always presented a problem, and various methods have been employed in order to obtain satisfactory slides showing reasonable numbers of cells. The majority of workers use Papanicolaou's method (Papanicolaou, 1954), in which a centrifuged sediment of urine is mixed with albumin, smeared on to glass slides previously coated with an albumin film, and fixed in an alcohol-ether solution. The smears are said to adhere better if they are allowed to dry at the edges before fixation and a coating of celloidin is applied before staining. Other workers have used substances other than albumin for adding to the sediment to promote adhesion, including pectin (Rofe, 1955), leuconostoc cultures and human saliva (Sagi and Mackenzie, 1958), and glycerinealbumin (Booth, 1959). Another method is the use of frosted glass slides (Dakin, 1955).

The purpose of this paper is to describe an entirely different method which is simple to use and gives preparations with excellent distribution of cells in adequate numbers. The basis of the method is the direct centrifugation of the cells on to a coverslip previously coated with an adhesive which retains its stickiness under water. The coverslip is held in a " perspex" cell containing the cell suspension and centrifuged with the sticky surface of the coverslip at right angles to the radius of the centrifuge. The apparatus used was originally designed as part of a technique for the differential separation of malignant cells from blood, but for various reasons has proved of little value for this purpose.

A special centrifuge head is an essential feature of the technique, and this will be described before recording the details of the method itself.

\section{Centrifuge Head}

This was designed to fit on to an M.S.E. "minor" centrifuge. Fig. 1 shows a part-sectioned elevation and plan views of the head, and Fig. 2 the head with

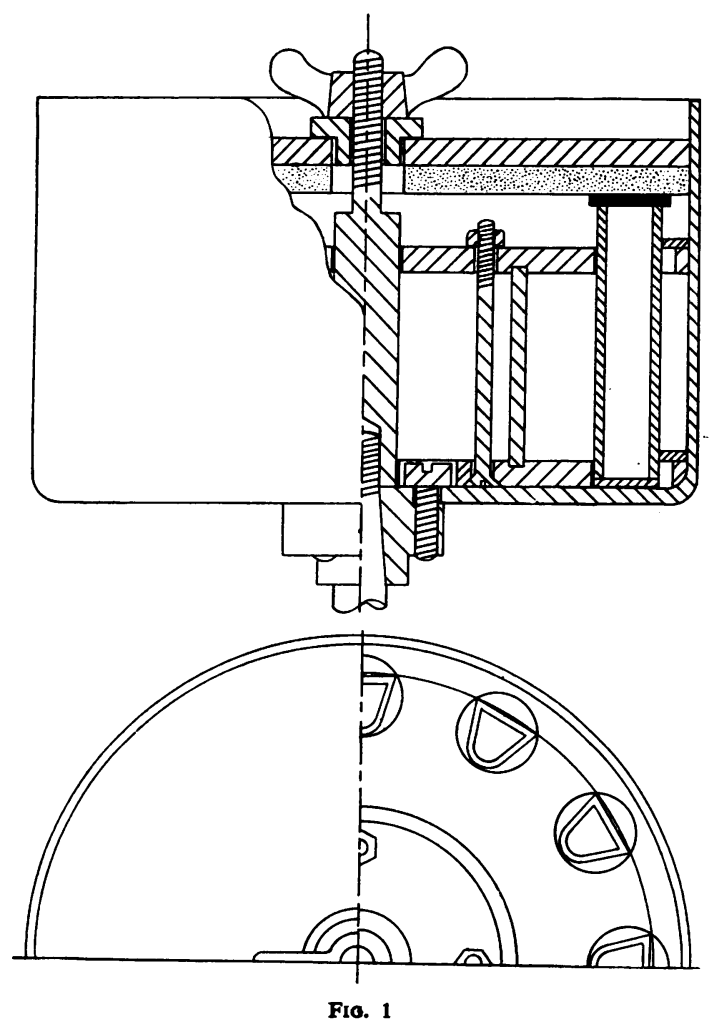




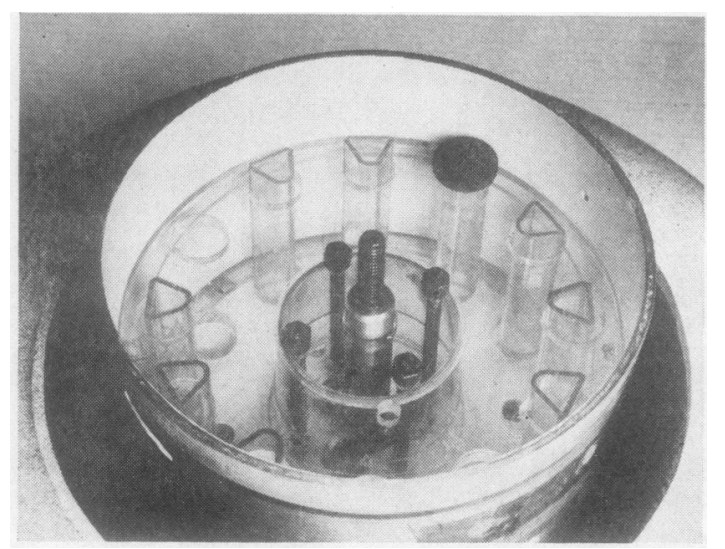

FIG. 2.-Centrifuge head with lid removed. Only one rubber washer is shown.

the lid removed. The bowl was made from an aluminium saucepan (of the deep-drawn, not cast, variety) to which a brass boss was fitted having a tapered bore to suit the centrifuge spindle. A "perspex" framework was fitted into the bowl to hold 12 specially designed centrifuge tubes. This consists of two $\frac{1}{4}$-in. thick discs separated by a length of $2 \frac{1}{2}$-in. diameter " perspex" tubing, fixed together to form a single unit by four bolts. Each of the discs has $12 \frac{7}{8}$-in. diameter holes equally spaced around the periphery, leaving a margin of $3 / 16$ in. between their

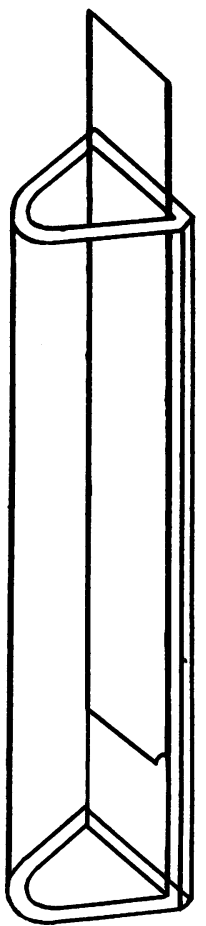
outer edges and the edges of the discs. A ring of $1 / 16$ in. thick "perspex," $5 / 16$ in. wide, is cemented to the outer part of the discs so that it partly intersects the holes, converting each of them into a D-shape. The centrifuge tubes are triangular in section and also made of " perspex." Details of one of these are shown in Fig. 3. The dimensions of the tubes provide that the flat vertical surface opposite the rounded corner takes a $2 \frac{1}{2}$ in. $x$ $\frac{1}{2}$ in. coverslip as a loose sliding fit when inserted as shown in the drawing. These tubes fit loosely into the holes in the "perspex" framework as shown in Figs. 1 and 2 . The centrifugal force keeps them in contact with the narrow peripheral ring, which ensures that the surfaces of the coverslips are accurately held at right angles to the radius of the head. The open ends of the tubes

$\overline{\text { FIG. 3.- "Perspex" cell with coverslip partly }}$ inserted. are sealed during centrifuging by individual circular rubber washers held in position by a separate $t$-in. thick " perspex" disc clamped down by a single wing nut on to the threaded rod fitted to the central spindle. A foam rubber pad glued to the under side of this disc gives even pressure on all the tubes.

\section{Method}

Preparation of Coverslips.-Rectangular No. 2 coverslips measuring $2 \frac{1}{2}$ in. $\times \frac{1}{2}$ in. are used. A small portion of one corner of each coverslip is snapped off (see Fig. 3) in order to facilitate removal from the "perspex" cell. The coverslips are thoroughly washed with soap and warm water, rinsed in water, and dried on a clean cotton cloth.

The adhesive used is "evostick" impact adhesive (PSM 317), which is obtained as a thick syrupy fluid and must be diluted $1: 5$ in benzene. The coverslips are coated on one surface with this solution, applying it fairly liberally with a camel-hair paintbrush and allowing them to dry in a dust-proof container. They will remain tacky indefinitely. If the adhesive is applied so that the snapped-off corner is always in the same position, this notch also serves to identify the adhesive side of the coverslip during fixing, staining, and mounting.

The prepared coverslips are placed in the $\overrightarrow{0}$ " perspex" centrifuge tubes with the broken cornerso 0 to the bottom of the tubes and the adhesive surfaces $\leq$ facing the rounded sides of the tubes. The triangularo음 section of the tube prevents the coverslip from falling away from the outer wall before centrifuging. The tubes are then ready to receive the cell suspension.

Preparation of Cell Suspension.-Most specimens of urine contain a mucoid substance which will prevent the cells from sticking to the adhesive coverslips, and this is first of all reduced to a negligible amount by a simple washing process.

The urine sample is spun in any suitable centrifuge tubes at 1,500 r.p.m. for five minutes. The supernatant fluid is sucked off and replaced by an equal volume of normal saline. The sediment is well mixed with the saline by inversion and then spun again as before. The supernatant is again removed and a further volume of saline added to the sediment and the two well mixed. The volume of the last addition of saline may vary within wide limits; it should theoretically be such that after preparation of the slide the distribution and number of the cells are ideal for ease of scanning and cell recognition, and obviously depend on the concentration of cells in the original sample. In practice, however, it has been found that satisfactory preparations are obtained from most urine samples if the volume of the final saline addition is equal to about one-tenth of the original volume of urine. The "perspex" tubes hold about $6 \mathrm{ml}$. of fluid, and this amount must therefore be a minimum. If the specimen is from a woman for examination of anterior urethral cells an original: final volume ratio of between $\frac{1}{2}$ and 1 
has been found satisfactory. After mixing the sediment with the final volume of saline, the suspension is transferred to the triangular plastic tubes which already have the adhesive coverslips in position and placed in the special centrifuge head. The individual rubber washers are placed over the open ends of the tube and the lid screwed firmly into position. The tubes are spun at 3,000 r.p.m. for 10 minutes. The coverslips are then removed from the tubes by means of a straight stiff wire with a small right-angled hook at one end which is passed to the bottom of the tube and hooked on to the broken corner of the coverslip. They are then immediately placed in the fixative.

Fixation, Staining, and Mounting.-Because the cells are adhering to an adhesive which is easily removed by organic solvents, fixatives and mounting media containing ether or xylol or having a high alcohol content must be avoided, and certain modifications of the common methods are used. Any watery fixative, such as formal saline or formal sublimate, can be used, but the best results were obtained using a modified Schaudinn's fixative with a lowered alcohol content, prepared by mixing equal quantities of absolute ethyl alcohol and a saturated aqueous solution of mercuric chloride. The adhesive is not attacked by this fixative provided the coverslips are left in it for two minutes only. The cells are well fixed in this time.

For routine work we use haemalum and eosin for staining. Papanicolaou's stains cannot be used owing to their high alcohol content. For studies of the female anterior urethra Shorr's stain is satisfactory. The counterstaining portion of Shorr's stain contains $50 \%$ alcohol, and the coverslips must not be left immersed for more than two minutes.

The common xylol- or turpentine-containing mounting media cannot be used alone because they dissolve the adhesive and produce clumping and distortion of the cells. Other media, such as polystyrene in mesitylene, do not appear to affect the adhesive in the same way as xylol but produce a haziness in the preparation together with some cell shrinkage. After trying various media it was found that a watery medium followed by colophonium in turpentine gave excellent results.

Following the final wash after staining, the coverslip is immediately flooded with a $15 \%$ solution of polyvinyl pyrrolidone (P.V.P.), drained, and allowed to dry with the adhesive surface uppermost. When dry the coverslip is mounted on a $3 \times 1$ in. slide with xylol-colophonium in the usual way. The acid dyes tend to be removed from the cells when the P.V.P. solution is added, but this does not occur if the solution is made slightly acid by the addition of acetic acid to $p \mathrm{H} \mathrm{5-6.}$

\section{Results}

The method has given excellent results in examination of urine in cases of carcinoma of the bladder. Whether cytological examination of urine for cancer cells is a worth-while process in view of the ease of cystoscopy and biopsy in hospital practice is a matter for debate. The method may perhaps be more useful in routine examination of urines from aniline dye workers. A useful application is for the examination of female urine for studies of hormone effects on the squame cells of the anterior urethra. These cells show parallel changes to those of the vagina (Brit. med. J., 1959) and provide an easily obtained source of material which can be taken by the subject herself as often as necessary. For this purpose slides are required containing large numbers of cells with a good distribution, and this has formerly been difficult to obtain for the reasons already mentioned.

It is often necessary to use urine as a sample in the preparation of prostatic cell smears, using the first few millimetres passed after massage. In this case the natural adhesive property of the prostatic secretion is greatly diminished and the adhesive slide method may be used to advantage.

The method also gives good preparations from pleural fluids. Some specimens have quite a high

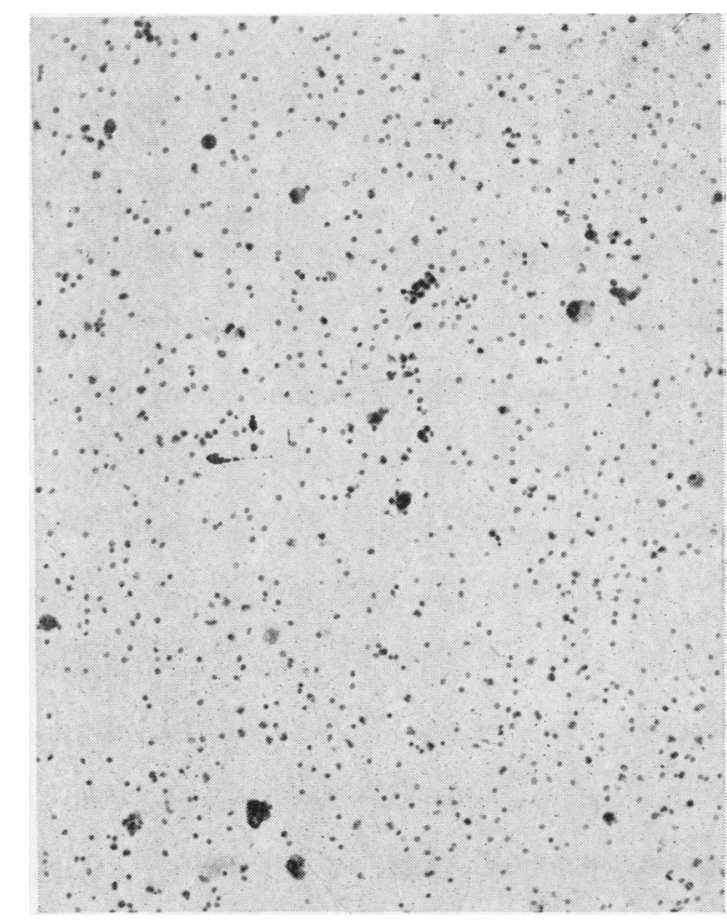

Fig. 4.-Urine preparation from case of carcinoma of bladder. $\times 150$. 


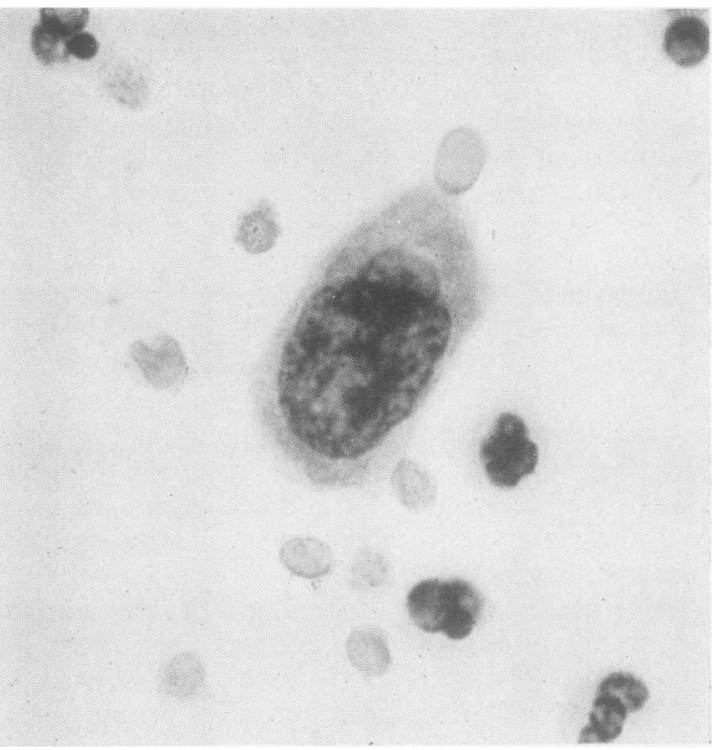

Fig. 5.-Malignant cell in urine from case of carcinoma of bladder. $\times 2,000$.

protein content and may clot, in which case it is best to remove the clot after it has contracted and to treat the remaining fluid as described for urine.

Figs. 4-6 show examples of the preparations obtained.

Fig. 4 is a low-power view of a preparation made from urine of a patient with a known carcinoma of the bladder and stained with haemalum and eosin. The field included in the picture represents $\frac{1}{2,500}$ of the area of the coverslip, and it is stressed that this distribution and cell density are of the same order over the whole area of the slide. In addition, the results are consistent, whereas the usual albumin technique gives inconsistent results with varying numbers of cells on different slides prepared from the same specimen, with a very irregular distribution of cells which makes scanning awkward.

Fig. 5 shows a malignant cell from a known case of bladder cancer and is included to show the degree of nuclear detail obtained with the method.

Fig. 6 shows a high-power view of anterior urethral cells prepared from normal female urine on the 25th day of a 32-day menstrual cycle and stained with Shorr's stain. The degree of cornification, nuclear variation, and curling of the cell margins are apprehended with the same ease (or difficulty) as with smears made in the usual way, but counting the number of cells is simplified by their regular distribution.

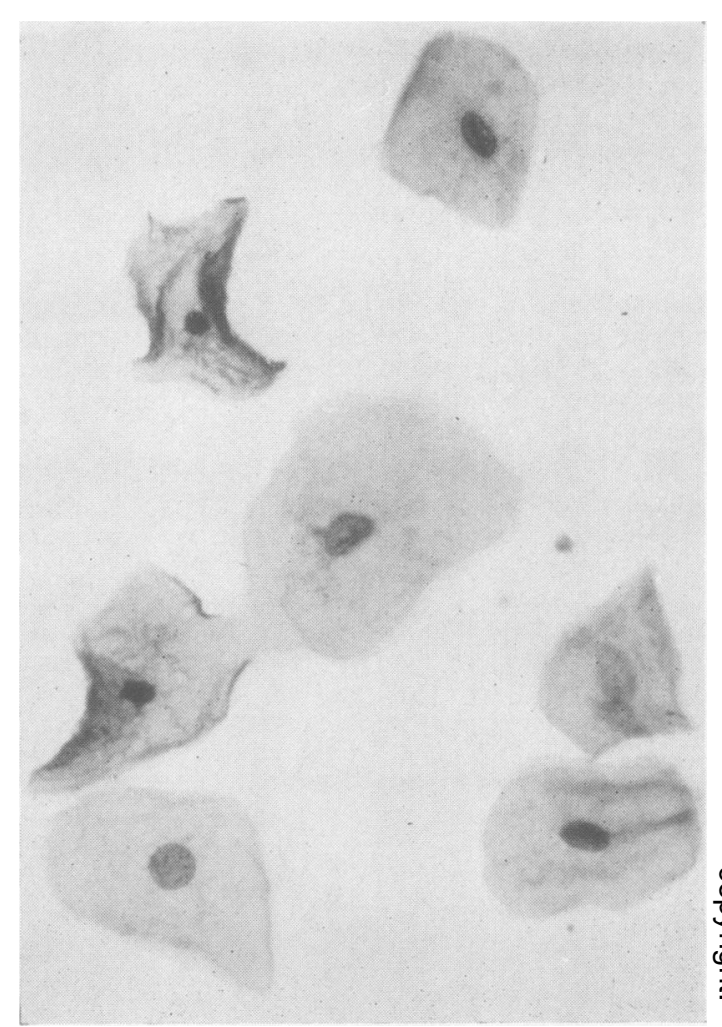

FIG. 6.-Urine preparation from normal female. $\times 600$.

\section{Summary}

A method of preparing specimens for cytology from protein-free fluids is described wherein the cells are directly centrifuged on to adhesive-coated slides. The method has great advantages over the usual process in that the number of cells retained on the slide is greatly increased together with a very even distribution.

I am grateful to Professor H. A. Magnus for his encouragement and helpful criticism in the preparation of this paper; also to Mr. G. A. Harwood for much technical advice, labour, and enthusiasm, and to Miss M. T. Pickett, research assistant in the Department of Endocrinology, Chelsea Hospital for Women, for supplying preparations of anterior urethral cells.

Messrs. Evode Ltd., of Stafford, kindly supplied the adhesive material used in this method.

\section{REFERENCES}

Booth, E. (1959). J. med. Lab. Technol., 16, 123 British Medical Journal (1959). Leading Article, 1, 1518

Dakin, E. S. (1955). Science, 121, 474.

Papanicolaou, G. N. (1954). Atlas of Exfoliative Cytology, p. 11. Combridge, Mass. Rofe, P. (1955). J. clin. Path., 8, 25.

Sagi, E. S., and Mackenzie, L. L. (1958). Cancer Cytol., 1, 24. 